\title{
Endoscopic Assisted Microscopic Skull Base Surgery
}

\author{
Mohammed Attia, Islam Alaghoy, Magdy El Hawary, Maamon Abo-Shosha \\ Skull-Base Group, Neurosurgery Department, Faculty of Medicine, Al-Azhar University, Cairo, Egypt \\ Email: i_aghory@hotmail.com
}

How to cite this paper: Attia, M., Alaghoy, I., El Hawary, M. and Abo-Shosha, M. (2018) Endoscopic Assisted Microscopic Skull Base Surgery. Open Journal of Modern Neurosurgery, 8, 187-200. https://doi.org/10.4236/ojmn.2018.82016

Received: February 7, 2018

Accepted: April 8, 2018

Published: April 11, 2018

Copyright (๑) 2018 by authors and Scientific Research Publishing Inc. This work is licensed under the Creative Commons Attribution International License (CC BY 4.0).

http://creativecommons.org/licenses/by/4.0/ (c) (i) Open Access

\begin{abstract}
Background: Skull base tumors presented great challenge for neurosurgeons since decades due to their deep location, associated morbidity and limitation of operative field; however modern neurosurgery using the endoscope and/or the microscope served in minimizing peri-operative morbidities and improving the clinical outcome. Objective: To demonstrate the value of endoscope-assisted microsurgical technique for resection of skull base tumors. $\mathrm{Pa}$ tients and Methods: 30 patients divided into 3 groups (10 patients had medial sphenoid wing meningioma constituted group 1, 10 patients had suprasellar meningioma constituted group 2 and 10 patients had Cerbello-Pontine Angle (CPA) epidermoids constituted group 3) were operated through Endoscope Assisted Microscopic Skull Base Surgery technique at Al-Azhar University Hospitals during the period starting from January 2016 till the end of September 2017 using a rigid endoscope for inspection of tumor boundaries and neighboring vascularity in addition to confirm the extent of resection. Tumor resection was tried in all cases. Intra-operative resection rate and post-operative radiological outcomes were assessed. Results: Total gross resection was possible in 27 patients (90\%). Subtotal resection was done in the other 3 cases (recurrent medial sphenoid wing meningiomas) due to excessive bleeding and adhesions of the tumors with vascular structures. Total resection of the tumor in post operative radiology was obtained in 24 patients (80\%) and post-operative residual tumor was noticed in 6 patients in early post operative radiology and in only 3 cases at 3 months follow up radiology. Conclusion: Endoscopic-assisted microsurgical approach is a reliable, safe and effective option for adequate surgical resection of skull base tumors. The technique allowed proper inspection of the tumor relations and vascularity, detection of any residual portions, providing better chance for gross total resection with minimal tissue damage or vascular injury as well as convenient clinical outcome.
\end{abstract}




\section{Keywords}

Skull Base, Endoscopic Assisted

\section{Introduction}

Until the beginning of the 20th century, lesions located at the base of the skull were largely inoperable. The base of the skull is a complex area that houses the main neural and vascular structures; so, it needs specific approaches to deal with. Skull base surgery involves the principle of drilling the skull base while avoiding major trauma and retraction to the brain [1]. The endoscope has become a widespread supplement to traditional skull base techniques, whether used in addition to the microscope or as the only visualizing tool. It provides a panoramic multi-angled view of the entire operative field [2]. The choice of approach is dependent on the goals of the operation (total versus subtotal resection), the areas of invasion, major arteries involved, and access to the tumor blood supply. Previous treatments, including surgery and radiation therapy, can affect the choice of surgical approach from the perspective of wound healing and surgical scar from a certain trajectory. Surgical complications may be nerve-related, cosmetic, and wound-related, including CSF leakage and vascular complications. The majority of these complications may be avoided by mastery of the surgical anatomy, preoperative planning, including appropriate structural and vascular imaging, intraoperative monitoring, and meticulous attention to detail at every stage of the operation. Vascular complications are likely the most feared complications of skull base approaches. They may be related to tumor infiltration into circle of Willis vessels, technical error, and inadequate preparation (neuronavigation, micro-Doppler, etc.). Large tumors related to the base of skull vessels should be evaluated preoperatively with cerebral angiography to assess for collateral circulation and test-occlusion when indicated [3]. Medial sphenoid wing meningiomas are considered one of the most challenging diseases to treat because of failure of total removal, high surgical mortality and morbidity rates, and a high rate of recurrence. Observation with radiological follow-up is preferable for asymptomatic and small tumors in elderly patients. For young patients, surgery at the time of tumor diagnosis, regardless of the size, even in incidental tumors, should be considered. Subtotal resection followed by adjuvant radiation therapy (RT) must be considered in medial sphenoid wing meningiomas with significant adherence or invasion of the internal carotid artery (ICA), middle cerebral artery (MCA), anterior cerebral artery (ACA), optic nerve, or cavernous sinus. The frontotemporal approach is used [4]. The cerebellopontine angle $(C P A)$ is a triangular space located posterior to the pyramid, inferior to the tentorium, lateral to the pons, and ventral to the cerebellum [5]. Tumors of the CPA account for 5\% - 10\% of all intracranial neoplasms [6]. Vestibular schwannomas (VSs) are the most common CPA tumor and account for $80 \%$ - 
$94 \%$ of them, followed by meningiomas (3\% - 10\% of CPA tumors) and the epidermoids $(2 \%-4 \%)$ [7]. The approaches to the CPA are either posterior (through the posterior cranial fossa) or lateral (through the petrous bone). The most popular approach is retrosigmoid suboccipital [8]. Suprasellar meningiomas originate most frequently from diaphragm sellae or anterior clinoid region [9]. Visual symptoms are still the most common presenting symptoms on admission, and the most common first symptom followed by headache [10]. Endoscope-assisted microsurgery has shown to be particularly useful in the treatment of expansive lesions located in the anterolateral cisterns of the skull base and in the cerebellopontine cisterns. Endoscope Assisted Microscopic Surgery (EAM) provides visualization of critical deep-seated neurovascular structures, allowing them to be dissected at an increased level of safety and preventing the superficial sectors from being exposed to inadvertent manipulation. EAM may be used effectively in the treatment of tumors located in the cerebellopontine angle (vestibular schwannomas, petroclival meningiomas, epidermoids, lower cranial nerve schwannomas, etc.) allowing visualization of critical neurovascular structures obscured by the lesion itself [11]. In the present study, we present our experience with endoscope-assisted microsurgical resection of skull base tumors through EAM approach in patients with medial sphenoid wing meningioma, CPA epidermoid and suprasellar meningioma regarding ability to achieve gross total resection and radiological outcome.

\section{Patients and Methods}

This study was conducted on 30 patients admitted and operated upon at Al-Azhar University Hospitals with a diagnosis of skull base tumor during the period starting from January 2016 till the end of September 2017. Skull base tumors involve all tumors that originate from the base of the skull starting from the frontal region anteriorly until the foramen magnum posteriorly. In this study, Selection of only 3 types of skull base tumors was done through the clinical picture of each group and the irradiological appearance at Computed Tomography (CT) scan and Magnetic Resonant Imaging (MRI). The study cases we redivided into 3 groups; Group 1 (10 patients with medial sphenoid wing meningioma), Group 2 (10 patients harbouring suprasellar meningioma) and Group 3 (10 patients with CPA epidermoid). All patients were subjected to full clinical assessment, including history, complete neurological examination. Radiological assessment was performed by CT and MRI with Gadolinium for the brain demarcating the extent and nature of the tumor. Full laboratory investigations were done for surgical preparation. No cerebral angiography was done in our series of cases since there was no encasement of large intracranial vessels in any case in this study (Table 1 , Table 2 ).

\subsection{Consent}

All patients in this study or their first degree relatives if uncooperative patients 
Table 1. Clinical and radiological data of the study patients.

\begin{tabular}{|c|c|c|c|c|}
\hline Case No. & Age (Ys) & Sex & Clinical Presentation & MRI findings \\
\hline 1 & 32 & Male & Headache, Rt 6th nerve palsy & Rt CPA epidermoid \\
\hline 2 & 45 & Female & Gradual progressive detrioration of vision in Rt eye & Rt Medial sphenoid wing meningioma \\
\hline 3 & 47 & Female & Bitemporal hemianopia & Suprasellar meningioma \\
\hline 4 & 59 & Male & Gradual progressive detrioration of vision in Rt eye & Rt Medial sphenoid wing meningioma \\
\hline 5 & 60 & Female & Bilateral visual deterioration, Headache & Suprasellar meningioma \\
\hline 6 & 23 & Female & Headache, Rt 6th nerve and Rt facial nerve palsy & Rt CPA epidermoid \\
\hline 7 & 57 & Female & $\begin{array}{l}\text { Gradual progressive detrioration of vision in Lt eye, } \\
\text { Headache }\end{array}$ & Recurrent Lt Medial sphenoid wing meningioma \\
\hline 8 & 62 & Male & Headache, Visual deterioration of Rt eye & Suprasellar meningioma \\
\hline 9 & 35 & Female & Rt sided weakness, headache & Lt CPA epidermoid \\
\hline 10 & 44 & Female & Headache, Visual deterioration of Lt eye & Suprasellar meningioma \\
\hline 11 & 25 & Male & $\begin{array}{l}\text { Gradual progressive detrioration of vision in Rt eye, } \\
\text { Headache }\end{array}$ & Rt Medial sphenoid wing meningioma \\
\hline 12 & 48 & Female & Headache, bilateral visual field defect & Suprasellar meningioma \\
\hline 13 & 59 & Male & Headache & Suprasellar meningioma \\
\hline 14 & 36 & Female & Headache, Lt trigeminal pain & Lt CPA epidermoid \\
\hline 15 & 57 & Female & $\begin{array}{l}\text { Gradual progressive detrioration of vision in Rt eye, } \\
\text { Headache }\end{array}$ & Rt Medial sphenoid wing meningioma \\
\hline 16 & 25 & Male & Bitemporal hemianopia & Suprasellar meningioma \\
\hline 17 & 66 & Female & Headache, Diminution of vision of Lt eye & Suprasellar meningioma \\
\hline 18 & 38 & Female & Headache, Tinnitus, Lt sided weakness & Rt CPA epidermoid \\
\hline 19 & 46 & Male & Lt 7th and 8th nerve palsy & Lt CPA epidermoid \\
\hline 20 & 58 & Female & Diminution of vision of Lt eye, Headache & Lt Medial sphenoid wing meningioma \\
\hline 21 & 36 & Female & Headache, Rt 6th nerve palsy & Rt CPA epidermoid \\
\hline 22 & 60 & Male & Diminution of vision of Rt eye & Suprasellar meningioma \\
\hline 23 & 50 & Female & $\begin{array}{l}\text { Gradual progressive detrioration of vision in Rt eye, } \\
\text { Headache }\end{array}$ & Recurrent Rt Medial sphenoid wing meningioma \\
\hline 24 & 40 & Male & Rt 7th and 8th nerve palsy & Rt CPA epidermoid \\
\hline 25 & 35 & Female & Bilateral visual field defect, Headache & Suprasellar meningioma \\
\hline 26 & 58 & Female & Visual field defect on Rt side & Rt Medial sphenoid wing meningioma \\
\hline 27 & 45 & Male & Rt sided weakness, Lt 7th nerve palsy & Lt CPA epidermoid \\
\hline 28 & 30 & Female & Gradual progressive deterioration of vision of Lt eye & Lt Medial sphenoid wing meningioma \\
\hline 29 & 29 & Male & Headache & Lt CPA epidermoid \\
\hline 30 & 56 & Female & Visual field defect on Rt side & Recurrent Rt Medial sphenoid wing meningioma \\
\hline
\end{tabular}


Table 2. Groups of the study patients and approach used in each group.

\begin{tabular}{cccc}
\hline Group & Diagnosis & Number of cases & Approach used \\
\hline Group 1 & Medial sphenoid wing meningioma & 10 & Pterional \\
Group 2 & Suprasellar meningioma & 10 & Pterional \\
Group 3 & CPA epidermoid & 10 & Retrosigmoid \\
\hline
\end{tabular}

signed a detailed consent about the procedure, its suspected benefits, its complications, available surgical alternatives and post-operative suspected lines of management.

\subsection{Surgical Technique}

Group 1 patients had medial sphenoid wing meningioma and were operated upon via pterional approach. Group 2 patients had suprasellar meningioma and were operated upon via pterional approach. Group 3 patients had CPA epidermoid tumors and were operated upon by retrosigmoid approach. Under general anesthesia and after injection of proper antiepileptic coverage in cases of suprasellar and medial sphenoid wing meningiomas, the endoscopic sheath was introduced and 0-degree rigid lensendoscope was placed for inspection of the tumor and surrounding areas under continuous irrigation with $37^{\circ}$ Cringer's solution providing clear visualization. Craniotomy was done for patients of Group 1 and Group 2 and craniectomy was done for patients of Group 3. The surgical corridor was opened by two retractors to visualize the tumor using a suitable piece of cottonoid for cortical protection. The operating microscope was then introduced with temporary getting the endoscope lens off field. Tumor debulking and removal was then started using suction and fine bipolar electrocautery with continuous irrigation. Two different angles of endoscope lenses $\left(0^{\circ}\right.$ and $30^{\circ}$ ) were used in several stages according to the degree of tumor accessibility to provide visualization of the feeding tumor vessels when microscopic visualization was insufficient. After the tumor mass was grossly excised and proper hemostasis was performed the endoscope was re-introduced to check the different anatomic corners where the tumor was located for the possibility of presence of any hidden residual which couldn't be detected until different angled scopes were used. The dura was then sutured. The bone was then repositioned and the wound closed in layers. Both intra-operative gross resection rate was assessed. Early and 3 months post operative radiology was done and post operative radiological resection rate was assessed.

\section{Case Presentation}

Case (1): Female patient, 38 years old, complained of headache, tinnitus, Rt facial pain and gradual progressive weakness of the Lt side of the body. MRI brain with contrast showed Rt CPA epidermoid tumor (Figure 1).

Case (2): Female patient, 58 years old, complained of headache and diminution 


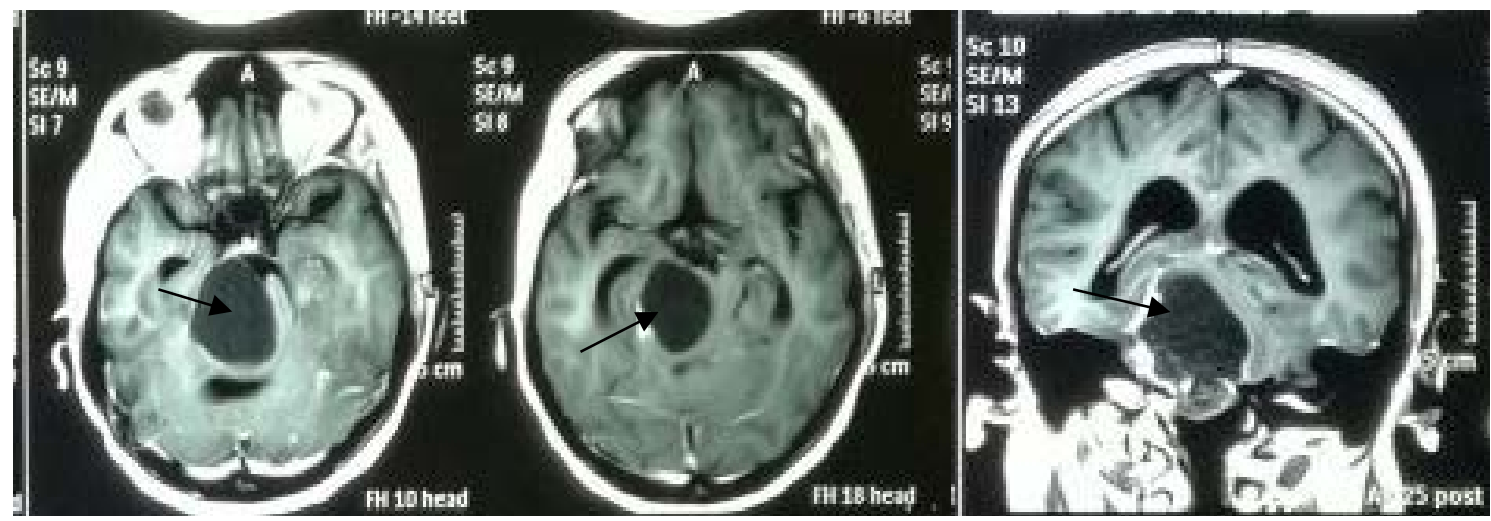

(a)

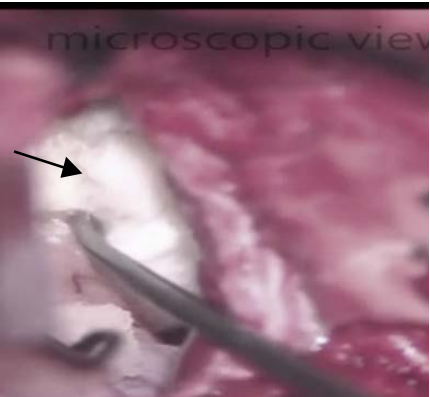

(b)

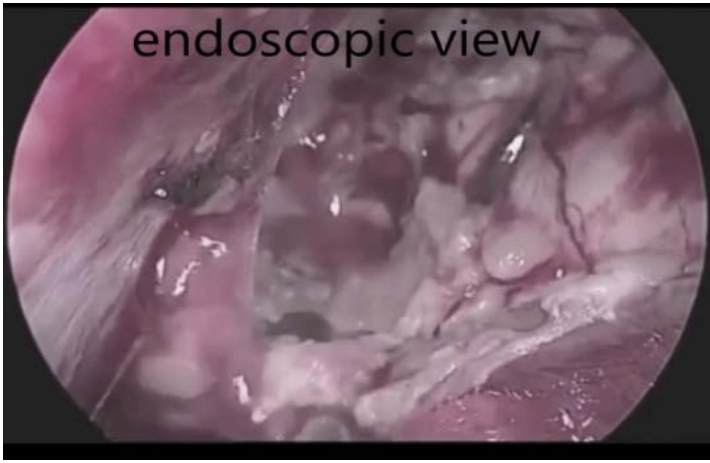

(e)

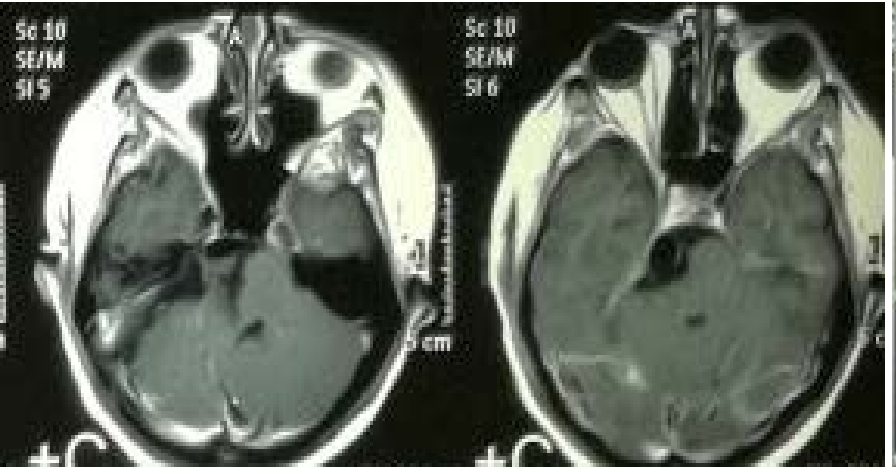

(g)

(c)

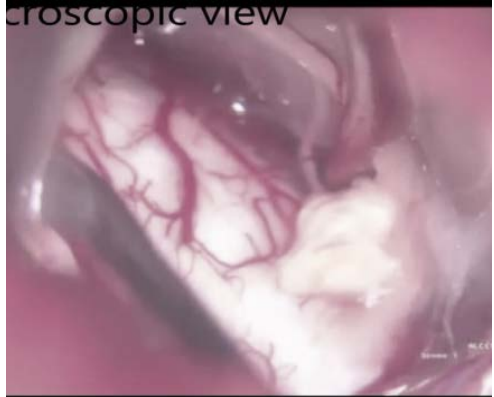

(d)



(f)
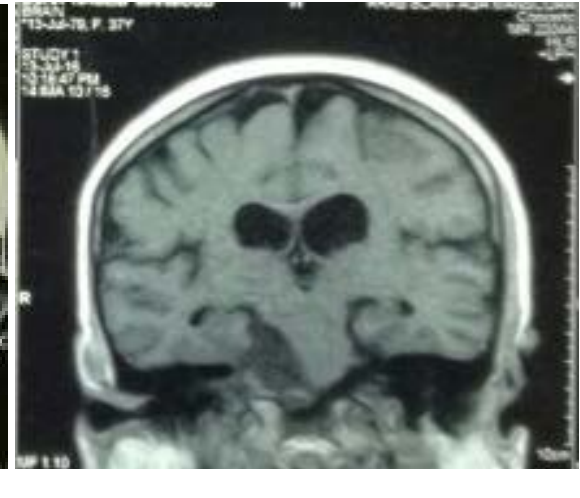

Figure 1. (a) MRI brain with contrast (axial and coronal views) showed right CPA epidermoid tumor (The arrowheads); (b) Microscopic view of dissection of the tumor (The arrowhead) in the CPA; (c) Microscopic view of excision of the tumor using the tumor forceps; (d) Microscopic view after microscopic excision of the tumor; (e) Angled Endoscopic view showing residual tumor; (f) Endoscopic view after total excision of the tumor using endoscope assisted microscopic surgery; (g) Post-operative MRI brain (axial and coronal) after excision of the mass showing small residual tumor. 
of vision of left eye, her visual Acuity: 6/60 on lt side, MRI brain with contrast showed lt medial sphenoid wing meningioma (Figure 2).

Case (3): Female patient, 60 years old, complained of headache and visual deterioration of both eyes, her visual acuity was $6 / 18$ on Rt side and hand motion on Lt side. MRI brain with contrast showed suprasellar meningioma (Figure 3).

\section{Results}

The study included 30 patients with a median age of 45.36 years (range: 23 - 66 years). They were 11 males (37\%) and 19 females (63\%). Medial Sphenoidal wing meningiomas were diagnosed in 8 females ( $80 \%$ of Group 1 patients) and in only 2 males (20\% of Group 1 patients). They occurred in patients with a median age of 49.5 years (range: 25 - 59 years). Suprasellar meningiomas occurred in 6 females (60\% of Group 2 patients) and in 4 males (40\% of Group 2 patients). The median age of Group 2 patients was 50.6 years (range: 25 - 66 years). CPA epidermoids were diagnosed in 5 females and 5 males (50\% of Group 3 patients for each). The median age of Group 3 patients was 36 years (range: 23 - 46 years). CPA epidermoids were noticed at more young age groups than other two

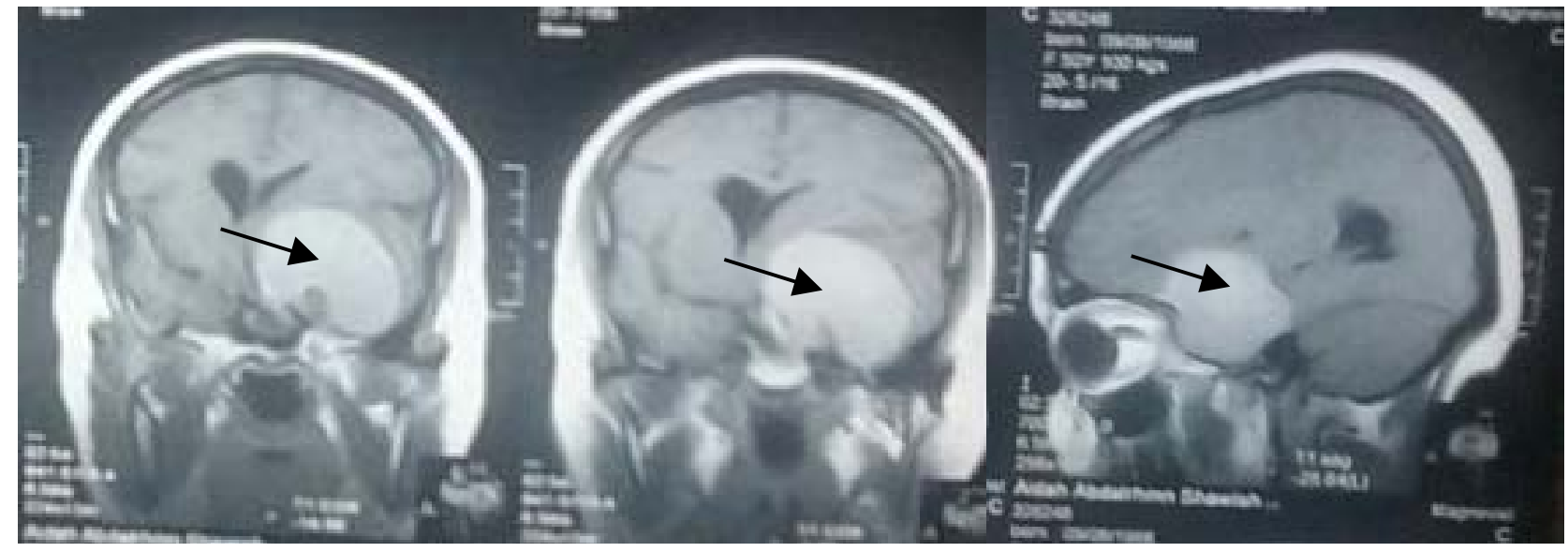

(a)

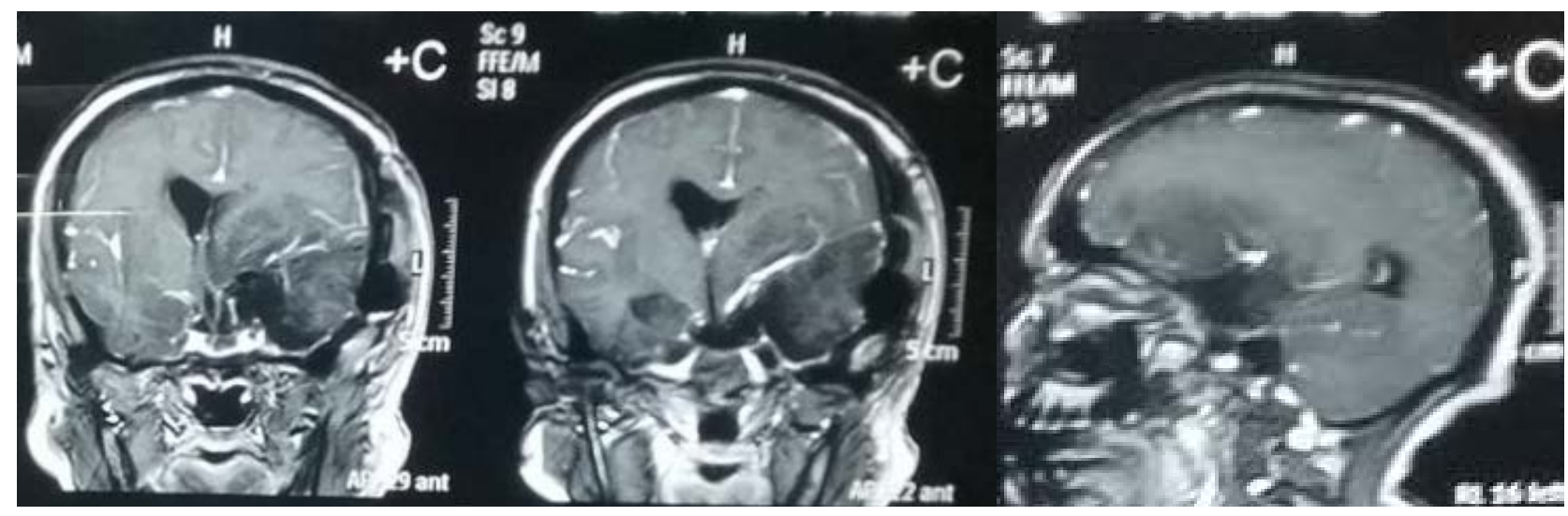

(b)

Figure 2. MRI brain with contrast (coronal and sagittal views) showed left medial sphenoid wing meningioma: (a) Pre-operative (The arrowheads); (b) Post-operative excision through endoscope assisted microscopic surgery. 

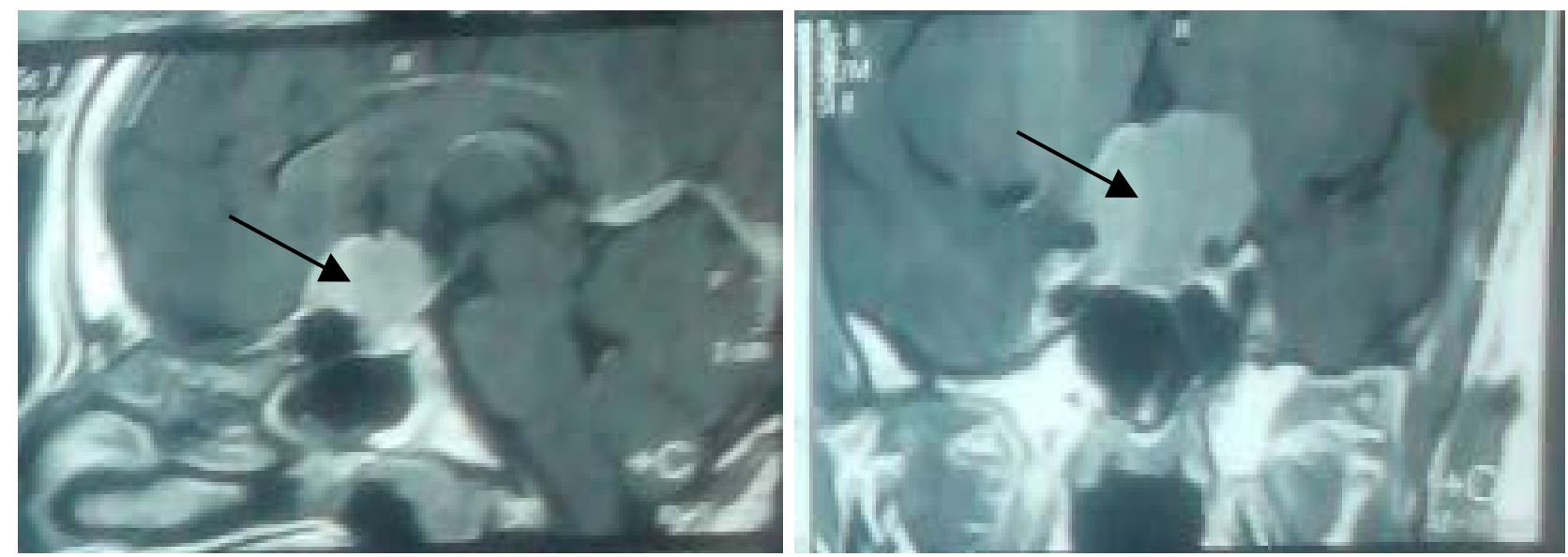

(a)
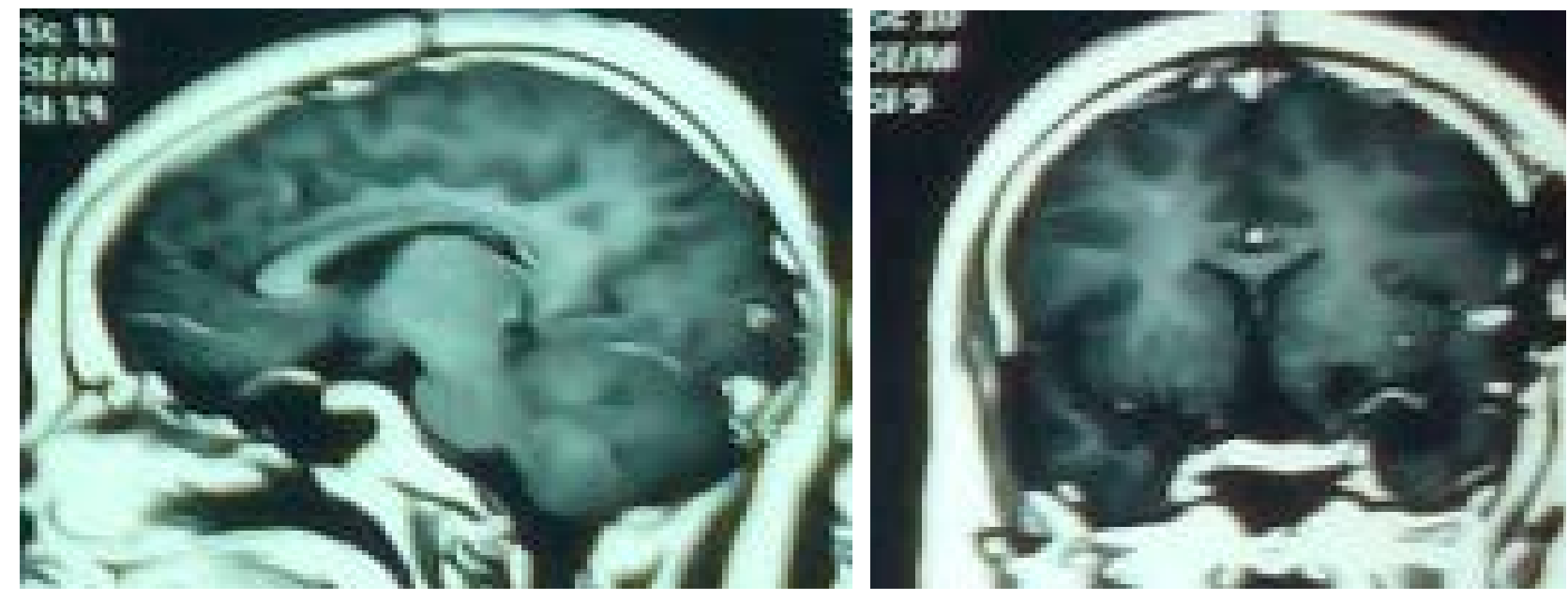

(b)

Figure 3. MRI brain with contrast (sagittal and coronal views) showed suprasellar meningioma: (a) Pre-operative (The arrowheads); (b) Post-operative excision through endoscope assisted microscopic surgery.

groups. Similarly, medial sphenoid wing meningiomas occurred predominantly at female patients than males.

\subsection{Intra-0perative Findings}

For Group 1 patients, introduction of the endoscope revealed possible residual following microsurgery in 4 patients (40\%), did not reveal any more residual following microsurgery in 3 patients (30\%) while total resection was not possible in the remaining 3 patients (30\%) who were operated for recurrent tumors even after introduction of the endoscope due to massive hemorrhage in two patients and due to adhesions between the tumor and neurovascular structures in the third one. For Group 2 patients, introduction of the endoscope revealed possible residual following microsurgery in 3 patients (30\%), did not reveal any more residual following microsurgery in 7 patients (70\%). For Group 3 patients, introduction of the endoscope revealed possible residual following microsurgery in all 10 cases $(100 \%)$ (Table 3$)$. 


\subsection{Gross Tumor Resection Rate Using Endoscope-Assisted Microsurgery (EAM)}

For Group 1, gross total resection was obtained in 7 patients (70\%), and was not possible in 3 patients (30\%). For Group 2, gross total resection was possible in all 10 patients (100\%). For Group 3, gross total resection of the tumor was possible in all 10 patients $(100 \%)$. In all study patients, the incidence of gross total resection of the tumors was $90 \%$ (Table 4 ).

\subsection{Radiological Resection Rate}

Total resection of the tumor in early postoperative radiology was obtained in 7 patients of $10(70 \%)$ of Group 1 patients, 10 patients of 10 (100\%) of Group 2 patients, in 7 patients of $10(70 \%)$ of Group 3 patients. This represents an overall percentage of $80 \%$ radiological resection rate of all study groups (Table 5).

Total resection of the tumor in 3-month follow up post operative radiology was obtained in 7 patients of $10(70 \%)$ of Group 1 patients, 10 patients of 10 (100\%) of Group 2 patients, in 10 patients of 10 (100\%) of Group 3 patients with overall percentage of $90 \%$ of all study patients. The disappearance of the tumor residual in 3 patients with $\mathrm{CPA}$ epidermoid may be due to filling of the space

Table 3. Intra-operative findings.

\begin{tabular}{ccccc}
\hline Group & $\begin{array}{c}\text { Number } \\
\text { of cases }\end{array}$ & $\begin{array}{c}\text { Total resection } \\
\text { using microscope } \\
\text { alone }\end{array}$ & $\begin{array}{c}\text { Total resection } \\
\text { after introduction } \\
\text { of the endoscope }\end{array}$ & $\begin{array}{c}\text { Residual tumor } \\
\text { after endoscope } \\
\text { assisted microsurgery }\end{array}$ \\
\hline 1 & 10 & 3 & 4 & 3 \\
3 & 10 & 7 & 3 & - \\
\hline
\end{tabular}

Table 4. Gross tumor resection rate using endoscope-assisted microsurgery.

\begin{tabular}{cccc}
\hline Group & Number of patients & Gross total resection & Residual tumor \\
\hline $\mathbf{1}$ & 10 & $7 / 10(70 \%)$ & $3 / 10(30 \%)$ \\
$\mathbf{3}$ & 10 & $10 / 10(100 \%)$ & - \\
All study patients & 10 & $10 / 10(100 \%)$ & $3 / 30(10 \%)$ \\
\hline
\end{tabular}

Table 5. Early radiological tumor resection rate using endoscope-assisted microsurgery.

\begin{tabular}{cccc}
\hline Group & Number of patients & $\begin{array}{c}\text { Early radiological } \\
\text { total resection }\end{array}$ & $\begin{array}{c}\text { Early radiological } \\
\text { residual tumor }\end{array}$ \\
\hline $\mathbf{1}$ & 10 & $7 / 10(70 \%)$ & $3 / 10(30 \%)$ \\
$\mathbf{3}$ & 10 & $10 / 10(100 \%)$ & - \\
All study patients & 10 & $7 / 10(70 \%)$ & $3 / 10(30 \%)$ \\
\hline
\end{tabular}


with CSF not with epidermoid residual because it disappeared at 3 months follow up. Otherwise, if residual, it would not disappear. So, diffusion MRI for epidermoid cases is mandatory to exclude residual (Table 6).

\subsection{Peri-Operative Complications}

Peri-operative complications occurred in 5 patients (17\%) of all study patients. Vascular injury occurred in 2 patients with recurrent medial sphenoid wing meningioma, control of bleeding occurred, surgery aborted due to severe brain edema in one patient and due to anesthetic causes in the other one, $2^{\text {nd }}$ sitting was done with near total resection of the tumor using endoscope-assisted microsurgery. Transient Facial nerve palsy had occurred in 2 patients with CPA epidermoid that improved within 2 weeks after surgery. 6th nerve palsy occurred in one patient with CPA epidermoid that improved within one month after surgery.

\section{Discussion}

Endoscope-assisted microsurgery is a practical indication for application of endoscopic technology. Endoscopic approach minimizes potential morbidity of approaching skull base tumors. This technique is usually associated with improved clinical outcome, shorter operating times, and decreased need for extensive surgical manipulation that may lead to greater morbidity [12]. The current study involved a heterogenous group of skull base tumors divided into 3 groups. Group 1 patients were 10 cases with medial sphenoid wing meningiomas, Group 2 patients were 10 cases with suprasellar meningiomas, and Group 3 patients were 10 cases with CPA epidermoids. In this study, endoscope-assisted microsurgery was performed. This technique allowed good microsurgical access assisted with excellent lighting and visualization through the endoscope. With a total gross resection in $90 \%$ of patients, the results of the current series are considered. Gross tumor resection rate was $70 \%$ in group 1 patients and was $100 \%$ in both group 2 and group 3 patients. Total resection of the tumor in early post operative radiology was obtained in $70 \%$ of Group 1 patients, $100 \%$ of Group 2 patients, in $70 \%$ of Group 3 patients and in $80 \%$ of all study patients. Total resection of the tumor in the 3-month follow up post operative radiology was obtained in $70 \%$ of Group 1 patients, $100 \%$ of Group 2 patients, in $100 \%$ of Group

Table 6. 3-month post-operative radiological tumor resection rate using endoscope-assisted microsurgery.

\begin{tabular}{cccc}
\hline Group & Number of patients & Gross total resection & Residual tumor \\
\hline 1 & 10 & $7 / 10(70 \%)$ & $3 / 10(30 \%)$ \\
2 & 10 & $10 / 10(100 \%)$ & - \\
3 & 10 & $10 / 10(100 \%)$ & - \\
All study patients & 30 & $27 / 30(90 \%)$ & $3 / 30(10 \%)$ \\
\hline
\end{tabular}


3 patients and in $90 \%$ of all study patients. Postoperative complications were encountered in 5 cases (17\%), all of them were temporary and controlled. Abolfotoh et al. study concluded that EAM provides simultaneous microscopic and endoscopic visualization and dissection techniques through skull base approaches to the CPA tumors and it overcomes some of the shortcomings of the endoscopic-assisted surgery, further extends the surgical field, and increases the radicality of tumor resection with good functional outcomes [13]. Presutti et al. study proved that combined microscopic/endoscopic procedures have proved to be effective in the treatment of petrous apex lesions, allowing less destructive approaches compared to exclusive microscopic procedures [14]. In our study, the use of endoscope was highly valuable in patients with CPA epidermoids and in patients with medial sphenoid wing meningioma and was less valuable in patients with suprasellar meningiomas using pterional approach where microsurgical resection did the job of complete tumor resection successfully in $70 \%$ of patients. Zhang et al. study [15] on 178 cases of medial sphenoid wing meningiomas revealed total resection of the tumor in 118 of 178 cases $(66.3 \%)$ compared to $70 \%$ in the present study, subtotal in 60 of $178(33.7 \%)$ at the time of initial surgery comparable to $30 \%$ in the present study which were recurrent cases with adhesions between the tumor and neurovascular structures. There were no serious surgical complications except 2 patients with ptosis in Zhang et al. study. Yang et al. [16] analyzed the clinical and radiological data of 53 patients with giant and medial sphenoid wing meningiomas that showed gross total resection in 44 patients (83\%). 11 patients $(21 \%)$ were found to have secondary injury of cranial nerves II, III, and IV, and nine patients got recovered during the long-term observing follow-up period. Temporary surgical complications of vascular lesions occurred after surgery, such as cerebral angiospasm, ischemia, and edema; 24 patients (45\%) appeared to have infarction and dyskinesia of limbs. The results of the present study using Endoscope assisted microsurgery showed no such complications of cranial nerve palsy, although temporary vascular injury occurred in 2 patients also in the present study. In cases of suprasellar meningiomas and other sellar and suprasellar lesions, the trans-sphenoial approach is more increasingly utilized than before. In the present study, the authors used the pterional approach for surgical resection of these lesions with total tumor resection in all 10 cases after the use of the endoscope-assisted microsurgical technique. Bander et al. study [17] showed that endoscopic endonasal approach provided equivalent rates of resection to transcranial approach with better visual results, less trauma to the brain, and fewer seizures. Gross-total tumor resection (Simpson Grade I) was achieved in $76 \%$ of the cases in the Koutourousiou et al. study [18] using the endonasal endoscopic approach. Lu et al. study [19] on 29 patients using transcranial interhemispheric approach showed total resection of the tumor in 25 patients (86\%) and 4 were of subtotal resection of tumor in the other 4 . Postoperative diabetes insipidus occurred in 2 patients (7\%), and 1 (3\%) was with electrolyte imbalance. Schroeder et al. [20] conducted 
a study on eight patients harboring an epidermoid tumor of the CPA that treated using an endoscope-assisted microsurgical technique. A retrosigmoid suboccipital approach was used in five patients and a pterional transsylvian approach was chosen in the remaining three. In four patients the lesion was resected microsurgically and the endoscope was used repeatedly to verify complete tumor removal, whereas most of the tumor mass was removed with the aid of an operating microscope in the other four. Tumor parts extending into other cranial compartments that were not visible through the microscope were removed under endoscopic view by using rigid rod-lens scopes with 30 and 70 degrees angles of view. All epidermoids were completely evacuated and the membranes were widely resected. Large tumors occupying both the middle and posterior cranial fossa were removed through a single small opening without enlarging the craniotomy. Permanent hearing loss and permanent hypacusis were observed in one patient each. One patient with facial and one with abducent nerve palsy recovered within 6 and 4 months, respectively. In the present study, transient facial nerve palsy occurred in 2 patients and tansient 6th nerve palsy occurred in one patient. A transient weakness of the chewing muscles was encountered in one patient in Schroeder et al. study. Postoperative magnetic resonance imaging revealed no residual tumor in any patient in Schroeder et al. study and in 3 patients in the early post operative radiological study and after 3 months there was no residual tumor in.

\section{Limitations}

There were some limitations in this study including dependency only on the type and site of the lesion to differentiate the groups of the study patients. Inside each group, there were no differentiating criteria like the size of the lesion, its extensions, presence of recurrence, adherence to surrounding vessels. The follow up period of 3 months post-surgical procedure is another limitation in this study. Follow up for longer periods after surgical excision may be indicated to obtain more accurate results.

\section{Conclusion}

Endoscopic-assisted microsurgical approach is a reliable, safe and effective option for adequate surgical resection of skull base tumors. The technique allowed proper inspection of the tumor relations and vascularity, detection of any residual portions, providing better chance for gross total resection with minimal tissue damage or vascular injury as well as convenient clinical outcome. It proved very useful for tumors that can insinuate themselves around corners between neurovascular structures and going to hidden pockets like epidermoids as concluded from our study and from others as Schroeder et al.

\section{References}

[1] Samii, M., Tatagiba, M. and Gerganov, V. (2011) Basic Principles of Skull Base Sur- 
gery. Youmans Neuroslogical Surgery, 116, 1267-1284. https://doi.org/10.1016/B978-1-4160-5316-3.00119-2

[2] Tatagiba, M., Matthies, C. and Samii, M. (1996) Microendoscopy of the Internal Auditory Canal in Vestibular Schwannoma Surgery. Neurosurgery, 38, 737-740. https://doi.org/10.1227/00006123-199604000-00021

[3] Maio, S.D. and Sekhar, L.N. (2012) Skull Base Approaches. In: Principles of Neurological Surgery. 3rd Edition, 667-679. https://doi.org/10.1016/B978-1-4377-0701-4.00043-9

[4] Landriel, F. and Black, P. (2012) Meningiomas. In: Principles of Neurological Surgery. 3rd Edition, 541-564. https://doi.org/10.1016/B978-1-4377-0701-4.00036-1

[5] Rhoton, Jr. A.L. (2000) The Cerebellopontine Angle and Posterior Fossa Cranial Nerves by the Retrosigmoid Approach. Neurosurgery, 47, S93-129. https://doi.org/10.1093/neurosurgery/47.3.S93

[6] De Monte, F. (1993) Neoplasms and Cranial Nerves of the Posterior Fossa. In: Barrow, D.L., Ed., Surgery of the Cranial Nerves of the Posterior Fossa. American Association of Neurological Surgeons, Park Ridge, 253-254.

[7] Bonneville, F., Savatovsky, J. and Chiras, J. (2007) Imaging of Cerebellopontine Angle Lesions: An Update. European Radiology, 17, 2908-2920. https://doi.org/10.1007/s00330-007-0680-4

[8] Samii, M. and Gerganov, V.M. (2008) Surgery of Extraaxial Tumors of the Skull Base. Neurosurgery, 62, 1153-1168. https://doi.org/10.1227/01.neu.0000333782.19682.76

[9] Cushing, H. and Eisenhardt, C. (1962) Meningiomas. Their Classification, Regional Behavior, Life History and Surgical End Results. Hafner, New York, 225-241.

[10] Gregorius, F.K., Hepler, R.S. and Stern, W.E. (1975) Loss and Recovery of Vision with Suprasellar Meningiomas. Journal of Neurosurgery, 42, 69-75. https://doi.org/10.3171/jns.1975.42.1.0069

[11] Galzio, R.J. and Tschabitscher, M. (2010) Endoscope-Assisted Microneurosurgery. Principles, Methodology and Applications. Endo: Press ${ }^{\mathrm{Tw}}$, Tuttlingen.

[12] Choudhri, O., Feroze, A.H., Nathan, J., Cheshier, S. and Guzman, R. (2014) Ventricular Endoscopy in the Pediatric Population: Review of Indications. Child's Nervous System, 30, 1625-1643. https://doi.org/10.1007/s00381-014-2502-8

[13] Abolfotoh, M., Bi, W.L., Hong, C.K., Almefty, K.K., Boskovitz, A., Dunn, I.F. and Al-Mefty, O. (2015) The Combined Microscopic-Endoscopic Technique for Radical Resection of Cerebellopontine Angle Tumors. Journal of Neurosurgery, 123, 1301-1311. https://doi.org/10.3171/2014.10.JNS141465

[14] Presutti, L., Alicandri-Ciufelli, M., Rubini, A., Gioacchini, F.M. and Marchioni, D. (2014) Combined Lateral Microscopic/Endoscopic Approaches to Petrous Apex Lesions: Pilot Clinical Experiences. The Annals of Otology, Rhinology, and Laryngology, 123, 550-559. https://doi.org/10.1177/0003489414525342

[15] Zhang, J., Shrestha, R., Cai, B.W., Zhou, P.Z., Li, Y.P. and Jiang, S. (2014) Management of Large Medial Sphenoid Wing Meningiomas: A Series of 178 Cases. Turkish Neurosurgery, 24, 664-671.

[16] Yang, J., Ma, S.C., Liu, Y.H., Wei, L., Zhang, C.Y., Qi, J.F. and Yu, C.J. (2013) Large and Giant Medial Sphenoid Wing Meningiomas Involving Vascular Structures: Clinical Features and Management Experience in 53 Patients. Chinese Medical Journal, 126, 4470-4476. 
[17] Bander, E.D., Singh, H., Ogilvie, C.B., Cusic, R.C., Pisapia, D.J., Tsiouris, A.J., Anand, V.K. and Schwartz, T.H. (2017) Endoscopic Endonasal versus Transcranial Approach to Tuberculum Sellae and Planum Sphenoidale Meningiomas in a Similar Cohort of Patients. Journal of Neurosurgery, 128, 40-48. https://doi.org/10.3171/2016.9.JNS16823

[18] Koutourousiou, M., Fernandez-Miranda, J.C., Stefko, S.T., Wang, E.W., Snyderman, C.H. and Gardner, P.A. (2014) Endoscopic Endonasal Surgery for Suprasellar Meningiomas: Experience with 75 Patients. Journal of Neurosurgery, 120, 1326-1339. https://doi.org/10.3171/2014.2.JNS13767

[19] Lu, Z.F., Cheng, X.B., Zhao, Y.G. and Shi, B.Z. (2013) Twenty-Nine Cases of Resection of Suprasellar Meningioma through Small Bone Window: An Interhemispheric Approach. Contemporary Oncology, 17, 525-529.

[20] Schroeder, H.W., Oertel, J. and Gaab, M.R. (2004) Endoscope-Assisted Microsurgical Resection of Epidermoid Tumors of the Cerebellopontine Angle. Journal of Neurosurgery, 101, 227-232. https://doi.org/10.3171/jns.2004.101.2.0227 